\title{
Estimation of Trunk Inclination by Means of an Inclinometer
}

\author{
Akihiko SEo $^{1}$, Masayuki KaKeHaShI ${ }^{2}$, Satoko Tsuru $^{2}$ and Fumitaka Yoshinaga ${ }^{1}$ \\ ${ }^{1}$ Department of Public Health and ${ }^{2}$ Institute of Health Sciences, Hiroshima University School of Medicine
}

\begin{abstract}
Estimation of Trunk Inclination by Means of an Inclinometer: Akihiko SEo, et al. Department of Public Health, Hiroshima University School of Medicine-In an effort to estimate trunk inclination by means of a simple inclinometer, a theoretical model analysis was carried out to clarify the relation between "true" trunk inclination and the inclinometer reading. The effects of inclinometer location on the trunk, working posture, and weight handied were also investigated experimentally. The theoretical model was based on work by Anderson et al. to estimate pelvic rotation from trunk inclination. The theoretical results indicated that the relation between trunk inclination and the inclinometer reading would change according to the location of the inclinometer. The curves were concave, straight, and convex when the inclinometers were fixed on the trunk at low, intermediate, and high levels, respectively. In the experimental study, twelve male subjects held 1,5 , and $10 \mathrm{~kg}$ boxes at $0,20,40$, and 60 $\mathrm{cm}$ from the floor in forward-bending and squatting postures. The inclinometers were fixed on the back at L4. Th12, and Th5. The relation between trunk inclination and the inclinometer reading in measurement data showed concave, straight, and convex curves at L4, Th12, and Th5, respectively, as the theoretical model analysis had indicated. At $L 4$ and Th5, however, the relation could be regarded as linear because the coefficients of determinations $\left(R^{2}\right)$ of the theoretical models calculated by the regression analyses agreed closely to those of the simple linear models. The difference between the forwardbending and squatting postures was large at L4, but small at Th12 and Th5. The effect of the weight handled was small and negligible in making these estimates.
\end{abstract}

(J Occup Health 1997; $39: 51-56$ )

Key words: Workload, Posture, Low back injury, Inclinometer, Theoretical model

Received Feb 27, 1995; Accepted July 26, 1996

Correspondence to: A. Seo, Department of Public Health, Hiroshima University School of Medicine, 1-2-3 Kasumi, Minami-ku, Hiroshima 734, Japan
For ergonomic evaluation of working conditions, it is important to have a clear idea of working postures at actual work places ${ }^{14}$. Observational and goniometric methods are often used to record working postures ${ }^{3-6)}$. Inclinometers are also used for simplified quantitative measurement ${ }^{7-10}$, but the location of an inclinometer on the body is problematic, usually depending on the purpose of the observation $^{7-9)}$.

To evaluate low back load, the inclinometer is usually fixed on the upper body of the subject to record the trunk inclination (angle between vertical and a line between the acromion and greater trochanter) and a special calibration curve must be developed because the inclinometer does not measure the trunk inclination directly. If the trunk were a rigid component, the estimation would be simple and easy because trunk inclination and the inclinometer reading would show a linear relation, but actual trunk inclination involves trunk bending and pelvic rotation ${ }^{11,12)}$. Most of the trunk bending occurs in the lower portion of the spinal column $^{13,14)}$, and pelvic rotation is reduced according to the trunk inclination ${ }^{15)}$. The actual relation between trunk inclination and the inclinometer reading should therefore be nonlinear. To what extent and under what conditions can the inclinometer calibration method be linear? This point has been insufficiently studied.

In this paper we first try to clarify the relation between trunk inclination and the inclinometer reading by using a theoretical model. We then present experimental data on the effects of inclinometer location, working posture, and the weight handled on this relation.

\section{Theoretical Study}

According to a statistical analysis by Anderson et al., pelvic rotation can be estimated by the following equation ${ }^{15)}$ :

$$
\begin{gathered}
S=-17.519-0.11863 T+0.22687 K+0.0011904 \\
T K+0.00499 T^{2}-0.000753 K^{2}
\end{gathered}
$$

where $K$ is knee angle, $S$ is sacral rotation (or 
pelvic rotation), and $T$ is torso rotation (or trunk inclination).

Trunk bending $(F)$ and the inclinometer reading $(I)$ are defined in terms of equation (1) as follows:

$$
\begin{aligned}
& F=T-S \\
& I=S+k F
\end{aligned}
$$

where $k$ is a location parameter for the inclinometer.

Figure 1 shows a graph for equations (1), (2) and (3) where $K$ and $k$ are fixed at $180 \mathrm{deg}$. and 1.5 , respectively. Parameter $k$ indicates the effect of inclinometer location, as shown in Fig. 2. A higher value for $k$ is obtained when the inclinometer is fixed higher on the trunk, because the effect of bending of the trunk is more cumulative as the inclinometer is fixed at a higher location. The relation between trunk inclination and the inclinometer reading is linear when $k$ is 1 ; otherwise a convex or concave relation is obtained.

Knee flexion induces the pelvis to rotate backwards, so that the inclinometer reading for a given degree of trunk inclination decreases the more the knee is bent, as shown in Fig. 3. In squatting, flexion at the knee and hip can be varied freely even though trunk inclination remains unchanged. This freedom in joint flexion can accordingly give rise to a wide variation in the relation between trunk inclination and the inclinometer reading.

\section{Experimental Study}

\section{Subjects and methods}

The subjects were twelve healthy males who had experienced no low back pain (mean $\pm S D$ of age: $23.5 \pm 0.9$ yrs; height: $172.3 \pm 5.2 \mathrm{~cm}$; weight: $64.6 \pm$ $7.3 \mathrm{~kg}$ ). The subjects wore tight clothes for easy identification of body segments. Inclinometers were fixed with a pair of suspenders and a waist belt on the mid back at L4, Th12, and Th5. The inclinometers detected inclination with respect to gravity by means of a magnetic resistance sensor ${ }^{10)}$. Each inclinometer was calibrated with a linear function empirically determined to minimize calibration error for that device. The output of each inclinometer was digitized with a 12-bit A-D converter (Micro science, DAS-1898XPC, Japan) at $20 \mathrm{~Hz}$
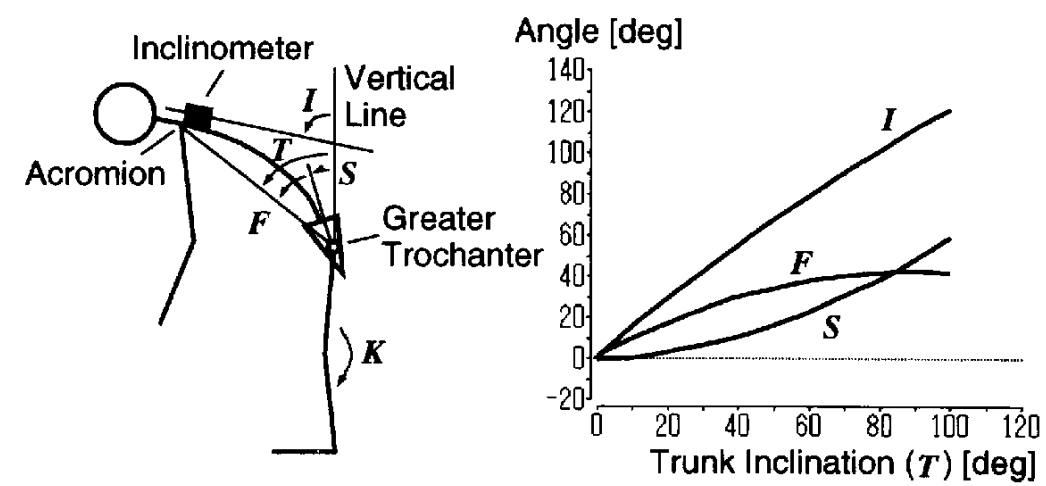

Fig. 1. Model and graph for pelvic rotation $(S)$, trunk bending $(F)$, and inclinometer reading $(I)$ as a function of trunk inclination $(T)$. To draw the lines, knee angle $(K)$ and the location parameter $(k)$ are fixed at $180 \mathrm{deg}$ and 1.5 , respectively.

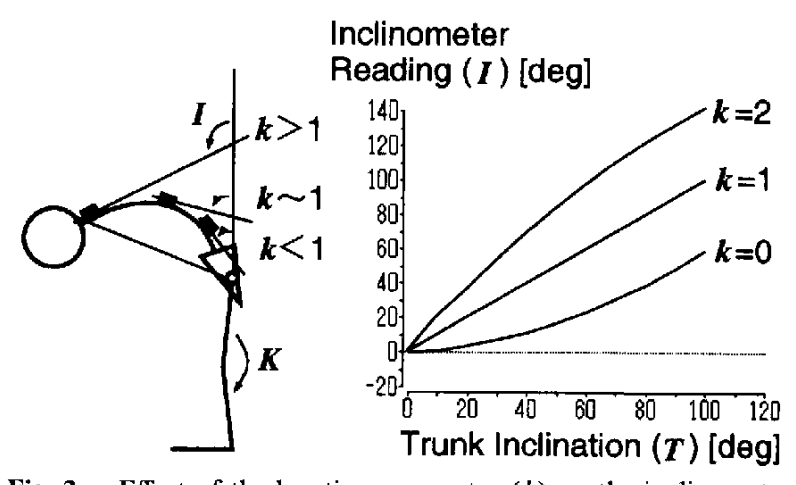

Fig. 2. Effect of the location parameter $(k)$ on the inclinometer reading $(I)$. To draw the lines, knee angle $(K)$ is fixed at $180 \mathrm{deg}$.



Fig. 3. Effect of the knee angle $(K)$ on pelvic rotation $(S)$, trunk bending $(F)$, and inclinometer reading $(I)$. To draw the lines, location parameter $k$ is fixed at 1.5 . 
and stored in a personal computer (Epson, PC-386 LS, Japan). Trunk inclination was measured photographically with the aid of a protractor. The locations of the acromion and greater trochanter were checked before the experiments and no markers were used to identify the locations.

For the experiment, the subjects held boxes at various heights in forward-bending and squatting postures. The boxes (size: $30 \times 35 \times 25 \mathrm{~cm}$ ) weighed 1,5 , and $10 \mathrm{~kg}$. The subjects held the boxes at heights of $0,20,40$, and $60 \mathrm{~cm}$ above the floor. In the forward-bending posture task, data were also obtained when the subjects held the boxes while standing upright. The subjects were ordered to flex their knees for the squatting posture, but the degree of trunk inclination and knee flexion was not specified. The conditions for posture, weight handled, and holding height were randomly assigned.

\section{Analysis}

The following four statistical models were used to test the validity and linearity of the theoretical model and to evaluate the effects of inclinometer location, posture, and weight handled on the relation between trunk inclination and the inclinometer reading:

theoretical full model

$$
I-S+k(T-S)+w W+\varepsilon
$$

theoretical reduced model

$$
I=S+k(T-S)+\varepsilon
$$

linear full model

$$
I=a T+b+w W+\varepsilon
$$

linear reduced model

$$
I=a T+b+\varepsilon
$$

where $a$ and $b$ are regression coefficients, $I$ is the inclinometer reading, $k$ is the location parameter for the inclinometer, $S$ is pelvic rotation calculated by equation (1), $T$ is trunk inclination, $W$ is weight handled, $w$ is weight parameter, and $\varepsilon$ is an error term with a normal distribution $\mathrm{N}\left(0, \sigma^{2}\right)$.

Equation (5) was a simple statistical model based on theoretical equation (3). Equation (4) was an expansion of equation (5) by adding in a weight factor. In equations (4) and (5), the knee angle $(K)$ was fixed at $180 \mathrm{deg}$ to calculate pelvic rotation (S). Stable solutions could not be obtained when both parameters $K$ and $k$ were included in the equation because the effects of $K$ and $k$ were mathematically similar, as shown in Figs. 2 and 3 . Parameter $k$ for the squatting posture thus reflected the effect of knee flexion in addition to inclinometer location.

Equations (6) and (7) were simple linear models to test the linearity of theoretical models (4) and (5). Equations (4) and (5) were nonlinear because $S$ was a quadratic function of $T$, as shown in Fig. 2 .
Equations (6) and (7) could be fit to the measured data when nonlinearity was not dominant.

Parameters $k, w, a$, and $b$ in equations (4) to (7) were calculated by a least squares method. To check the fitness of the model, the coefficient of determination $\left(R^{2}\right)$ and standard error (SE) for regression were calculated with the following equations:

$$
\begin{aligned}
& R^{2}=\sum_{i=1}^{N}\left(\hat{I}_{i}-\bar{I}\right)^{2} / \sum_{i=1}^{N}\left(I_{i}-\bar{I}\right)^{2} \\
& S E=\sqrt{\sum_{i=1}^{N}\left(I_{i}-\hat{I}_{i}\right)^{2} /\left(N-N_{p}\right)}
\end{aligned}
$$
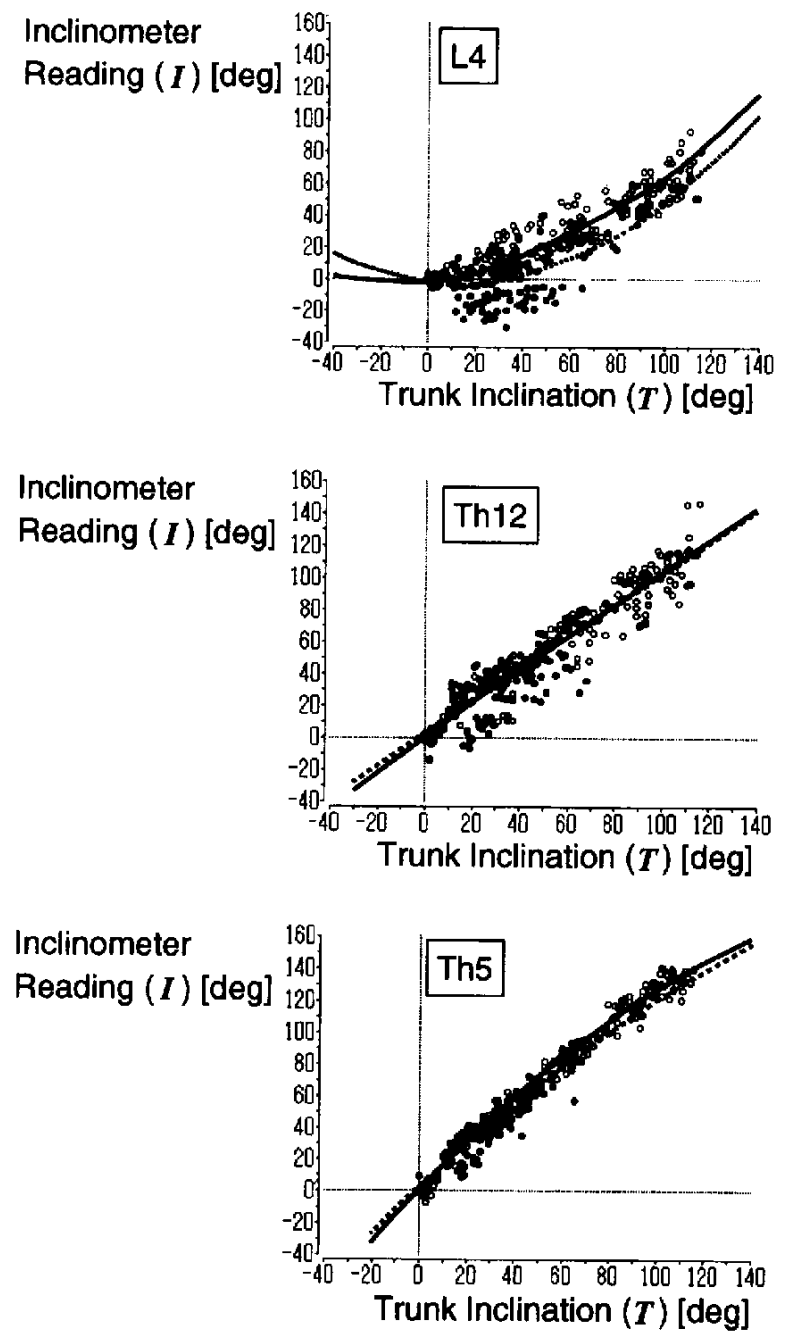

Fig. 4. Relation between trunk inclination ( $T$ ) and inclinometer reading $(I)$ at $\mathrm{L} 4, \mathrm{Th} 12$, and Th5. Open and closed circles indicate data for the forward-bending and squatting postures, respectively. Straight and dotted lines indicate the lines for the theoretical full models in the forward-bending and squatting postures, respectively. To draw the lines, knee angle $(K)$ and weight handled $(W)$ were fixed at $180 \mathrm{deg}$ and $5 \mathrm{~kg}$, respectively. 
Table 1. Summary of the model parameters, coefficients of determination $\left(R^{2}\right)$, and standard errors (SE) of inclinometer readings for four models

\begin{tabular}{|c|c|c|c|c|c|}
\hline Location & Posture & Model & Parameter & $R^{2}$ & $S E(\mathrm{deg})$ \\
\hline \multirow[t]{12}{*}{ L4 } & Forward-bending & Theoretical full model & $(k, w)=(0.0835,0.2511)$ & 0.825 & 9.82 \\
\hline & & Theoretical reduced model & $k=0.1201$ & 0.823 & 9.85 \\
\hline & & Linear full model & $(a, b, w)-(0.591,-1.915,0.025)$ & 0.867 & 8.57 \\
\hline & & Linear reduced model & $(a, b)=(0.591,-1.788)$ & 0.867 & 8.55 \\
\hline & Squatting & Theoretical full model & $(k, w)=(-0.2831,-0.0104)$ & 0.278 & 13.41 \\
\hline & & Theoretical reduced model & $k=-0.2851$ & 0.278 & 13.36 \\
\hline & & Linear full model & $(a, b, w)=(0.505,-17.645,0.299)$ & 0.283 & 13.42 \\
\hline & & Linear reduced model & $(a, b)-(0.505,-16.065)$ & 0.278 & 13.42 \\
\hline & Forward-bending & Theoretical full model & $(k, w)=(-0.0072,0.0675)$ & 0.743 & 12.97 \\
\hline & $t$ & Theoretical reduced model & $k=-0.0180$ & 0.743 & 12.95 \\
\hline & Squatting & Linear full model & $(a, b, w)=(0.679,-14.457,0.129)$ & 0.714 & 13.70 \\
\hline & & Linear reduced model & $(a, b)=(0.680,-13.783)$ & 0.713 & 13.69 \\
\hline \multirow[t]{12}{*}{ Th12 } & Forward-bending & Theoretical full model & $(k, w)-(1.1070,-0.1826)$ & 0.925 & 11.09 \\
\hline & & Theoretical reduced model & $k-1.0804$ & 0.925 & 11.08 \\
\hline & & Linear full model & $(a, b, w)-(1.056,-1.095,-0.050)$ & 0.925 & 11.12 \\
\hline & & Linear reduced madel & $(a, b)=(1.056,-1.353)$ & 0.925 & 11.09 \\
\hline & Squatting & Theoretical full model & $(k, w)=(0.9934,0.2666)$ & 0.599 & 12.51 \\
\hline & & Theoretical reduced model & $k=1.0436$ & 0.596 & 12.51 \\
\hline & & Linear full model & $(a, b, w)=(0.932,3.666,0.087)$ & 0.604 & 12.48 \\
\hline & & Linear reduced model & $(a, b)=(0.932,4.126)$ & 0.604 & 12.44 \\
\hline & Forward-bending & Theoretical full model & $(k, w)=(1.0701,-0.0141)$ & 0.887 & 11.75 \\
\hline & + & Theoretical reduced model & $k-1.0678$ & 0.887 & 11.73 \\
\hline & Squatting & Linear ful1 model & $(a, b, w)-(1.035,0.072,0.013)$ & 0.887 & 11.76 \\
\hline & & Linear reduced model & $(a, b)=(1.035,0.142)$ & 0.887 & 11.74 \\
\hline \multirow[t]{12}{*}{ Th5 } & Forward-bending & Theoretical full model & $(k, w)=(1.6460,-0.1649)$ & 0.985 & 5.77 \\
\hline & & Theoretical reduced model & $k=1.6220$ & 0.985 & 5.79 \\
\hline & & Linear full model & $(a, b, w)=(1.261,2.699,0.031)$ & 0.980 & 6.72 \\
\hline & & Linear reduced model & $(a, b)=(1.261,2.859)$ & 0.980 & 6.70 \\
\hline & Squatting & Theoretical full model & $(k, w)=(1.4336,0.2025)$ & 0.879 & 7.29 \\
\hline & & Theoretical reduced model & $k-1.4718$ & 0.877 & 7.32 \\
\hline & & Linear full model & $(a, b, w)=(1.195,4.812,0.118)$ & 0.883 & 7.21 \\
\hline & & Linear reduced model & $(a, b)=(1.195,5.436)$ & 0.882 & 7.20 \\
\hline & Forward-bending & Theoretical full model & $(k, w)=(1.5849,-0.0880)$ & 0.971 & 6.86 \\
\hline & + & Theoretical reduced model & $k=1.5708$ & 0.971 & 6.86 \\
\hline & Squatting & Linear full model & $(a, b, w)=(1.252,3.048,0.071)$ & 0.971 & 6.94 \\
\hline & & Linear reduced model & $(a, b)-(1.252,3.416)$ & 0.970 & 6.93 \\
\hline \multicolumn{6}{|c|}{ Model equations and parameters are as follows: } \\
\hline \multicolumn{2}{|c|}{ Theoretical full model } & $\begin{array}{l}I=S+k(T-S)+w \boldsymbol{W}+\varepsilon \\
I=S+k(T-S)+\varepsilon\end{array}$ & & & \\
\hline \multicolumn{2}{|c|}{$\begin{array}{l}\text { Theoretical reduced model } \\
\text { Linear full model }\end{array}$} & $I=a T+b+w W+\varepsilon$ & & & \\
\hline Linear $\mathrm{r}$ & uced model & $I=a T+b+\varepsilon$ & & & \\
\hline
\end{tabular}

where $I$ is inclinometer reading, $S$ is pelvic rotation, $T$ is trunk inclination, $W$ is weight handled, $a$ and $b$ are regression coefficients, $k$ is location parameter for the inclinometer, $w$ is weight parameter, and $\varepsilon$ is an error term with a normal distribution $N\left(0, \sigma^{2}\right)$. SE is an unbiased estimator of the $\sigma$ of $\varepsilon$.

where $I_{i}$ was the $i$ th inclinometer reading, $\hat{I}_{i}$ was the $i$ th value estimated with the model, $I$ was mean value, $N$ was number of data in the sample, and $N_{p}$ was number of parameters included in the model.

The model fits the data well when $R^{2}$ is closer to
1. $S E$ is an unbiased estimator of the $\sigma$ of $\varepsilon$ in equations (4) to (7), indicating the magnitude of the residuals between measured and estimated values. A model with the highest $R^{2}$ and lowest $S E$ is the best one statistically, but when the differences 
among models in $R^{2}$ and $S E$ are too small to be of practical concern, the best model is chosen from the standpoint of feasibility or usability.

The effect of the weight handled was included in the model as a continuous variable instead of as a categorical variable, as indicated in equations (4) and (6). In this way the unit for the coefficient $w$ was $\mathrm{deg} / \mathrm{kg}$, so the effect of the weight on the inclinometer reading was easy to interpret.

\section{Results}

Scatter diagrams and regression lines of the theoretical full models are shown in Fig. 4 and the results of the regression analyses are shown in Table 1.

In the forward-bending posture, the measured data for L4, Th12, and Th5 levels showed a tendency to be slightly concave, almost linear, and slightly convex, respectively, as Fig. 4 indicates. The curves for the theoretical full models matched the pattern for the measured data as $R^{2}$ was 0.8 or higher for any inclinometer location.

In the squatting posture, the measured data were scattered and showed no clearly consistent concave or convex pattern. The value for $R^{2}$ was lower and that for $S E$ was higher than those in the case of the forward-bending posture. For the two postures, at the level of L4, the values for inclination and the curve resulting from least-squares fit to the theoretical model perceptibly differed. At the Th12 and Th5 levels, on the other hand, these differences between forward-bending and squatting postures were so small that the $R^{2}$ and $S E$ for theoretical models pooled from data for both postures hardly differed from those calculated for either posture at Th12 and at $\mathrm{Th} 5$

Differences between full and reduced models and differences between the theoretical and linear models were small for all locations and postures, as Table 1 indicates.

\section{Discussion}

\section{Linearity of the relation between trunk inclina-} tion and inclinometer reading

The theoretical analysis indicated that the nonlinearity of the relation between trunk inclination and the inclinometer reading depended on the inclinometer location, as shown in Figs. 1 and 2. The nonlinearity could be neglected when the location parameter $k$ was close to 1 . Dependency of the nonlinearity on the inclinometer location was observed in the experimental study, as shown in Fig. 4. At Th12, the relation of the measured data could be regarded as linear and the parameter $k$ was very close to 1 , as Table 1 indicates. At L4 and Th12, the measured data showed slightly con- cave or convex patterns. The values for $R^{2}$ and $S E$ in the theoretical models were in close agreement to those of the linear models for all inclinometer locations. The relation could therefore be regarded as linear at all locations between L4 and Th5 in practical use.

\section{Effect of working postures}

In squatting, the pelvis rotates backwards in conjunction with flexion of the knee and hip joints ${ }^{15,16)}$. To compensate for the rotation, the trunk has to bend further forward to maintain a given trunk inclination than in the forward-bending posture. The relation between trunk inclination and the inclinometer reading in the squatting posture may therefore differ from that in the forward-bending posture. Furthermore, scatter of the measured data was greater in the squatting posture, presumably because of greater freedom for variation in knee and hip joint flexion than in the forward-bending posture. The experimental study showed a clear difference between the model equations for the two postures and a low $R^{2}$ for the squatting posture at L4. At Th12 and Th5, on the other hand, even though the $R^{2}$ for the squatting posture was a little lower, only a small difference was seen between the model equations, suggesting that the effect of working posture can be neglected when the inclinometer is fixed high on the trunk.

\section{Effect of handling weights}

Anderson et al. reported that prediction of pelvic rotation was not improved by taking into account the weight handled ${ }^{15)}$. Our results also showed that the inclinometer reading was hardly affected by the weight handled because the $R^{2}$ for the reduced model was almost equal to that for the full model. If the trunk were a purely elastic body, it might bend more according to the magnitude of the weight handled ${ }^{17)}$, but trunk muscles contract in response to the weight to maintain the same posture. In addition, the trunk is drawn backwards to balance moments when the handling materials are held in front of the body. This may also tend to cancel out the effect of any potential increase in the inclinometer readings due to the weight handled.

\section{Best location for the inclinometer}

The experimental study showed that the inclinometer should be fixed at a high location around Th5 for relatively precise measurement because $R^{2}$ was highest, $S E$ was lowest, and the effect of posture was lowest with the inclinometer at that location. A location higher than Th5 is not recommended because the nonlinear tendency is increasing, 
as shown in Fig. 2. The nonlinearity would introduce a systematic bias if a linear calibration line were used. A lower location around Th12 would be better than Th5 from the viewpoint of linearity. If most of the work is carried out in the forwardbending posture, the inclinometer may be fixed at any location on the trunk, because $R^{2}$ was high, exceeding 0.8 from L4 to Th5. In this case, the most important factor in deciding the inclinometer location for actual use would probably be whether the inclinometer fixed on the body disturbs the work or not.

Acknowledgments: We would like to thank Prof. Paul D. Andrew, Hiroshima University School of Medicine, for his advice and for revising the English of the manuscript.

\section{References}

1) Erdil M, Dickerson OB, Chaffin DB. Biomechanics of manual materials handling and low-back pain. In: Zenz C, ed. Occupational medicine. St. Louis: Mosby-Year Book, 1994: 239-257.

2) Chaffin DB, Andersson GBJ. Occupational Biomechanics. New York: John Wiley \& Sons, 1991.

3) Kant I, Notermans JHV, Borm PJA. Observations of working postures in garages using the Ovako Working Posture Analyzing System (OWAS) and consequent workload reduction recommendations. Ergonomics 1990; 33: 209-220.

4) Seo A, Kakehashi M, Tsuru S, Amran A, Paeng J, Yoshinaga F. Development of a system for analyzing working postures. Ind Health 1993; 31: 69-77.

5) Rowe PJ, Nicol AC, Kelly IG. Flexible goniometer computer system for the assessment of hip function. Clin Biomech 1989; 4: 68-72.

6) Marras WS, Fathallah FA, Miller RJ, Davis SW,
Mirka GA. Accuracy of a three-dimensional lumbar motion monitor for recording dynamic trunk motion characteristics. Int J Ind Ergonomics 1992; 9: 75-87.

7) Otun EO, Anderson AD. An inclinometric method for continuous measurement of sagittal movement of the lumbar spine. Ergonomics 1988; 31: 303-315.

8) Aarås A, Stranden E. Measurement of postural angles during work. Ergonomics 1988; 31: 935-944.

9) Gilad I, Chaffin DB, Woolley C. A technique for assessment of torso kinesiology. Appl Ergonomics 1989; 20: 82-88.

10) Seo A, Tsuru S, Kakehashi M, Yoshinaga F. A simple apparatus using inclinometer for monitoring working postures. Jpn J Ind Health 1994; 36: 406411.

1) Anderson CK, Chaffin DB, Herrin GD, Matthews LS. A biomechanical model of the lumbosacral joint during lifting activities. J Biomechanics 1985; 18: 571 -584 .

12) Garg A, Herrin GD. Stoop or squat: A biomechanical and metabolic evaluation. AIIE Transactions 1979; 11: 293-302.

13) Davis PR, Troup JDG, Burnard JH. Movements of the thoracic and lumbar spine when lifting: a chrono-cyclophotographic study. J Anat Lond 1965; 99: 13 $-26$.

14) Lindahl $O$. Determination of the sagittal mobility of the lumbar spine. Acta Orthop Scand 1966; 37: 241254.

15) Anderson CK, Chaffin DB, Herrin GD. A study of lumbosacral orientation under varied static loads. Spine 1986; 11: 456-462

16) Chaffin DB. A computerized biomechanical model: development of and use in studying gross body actions. J Biomechanics 1969; 2: 429-441.

17) Scholten PJM, Veldhuize AG. The bending stiffness of the trunk. Spine 1986; 11:463-467. 